\title{
Social class, happiness and environmentally friendly behavior Nan $\mathrm{Ye}^{1, \mathrm{a}}$, Xiao Zhang ${ }^{2, \mathrm{~b},{ }^{*}}$ and Hang $\mathrm{Yu}{ }^{3, \mathrm{c}}$ \\ 1,2,3 Business School of Jiangsu Normal University, Xuzhou, Jiangsu, China anye@jsnu.edu.cn, b244785316@qq.com, c1174607211@qq.com \\ ${ }^{*}$ Corresponding author
}

Key words: Social class; Happiness; Environmentally friendly behaviour; CGSS

\begin{abstract}
Based on the 2013 data of Chinese General Social Survey (CGSS), this paper analyzes the impact of subjective social class on environmental friendly behavior, and introduces the intermediary variable of the sense of happiness to explain the mechanism of the influence of subjective social stratum on environmentally friendly behavior. The result shows that social class positively affects pro-environmental behaviors, not only the environmentally friendly behaviors that are in the public domain but also that are in the private sector; Happiness has a positive influence on the environment friendly behavior in private domain, but a negative influence on the environment friendly behavior in public domain. Moreover, happiness plays a partial mediating role in the influence of social class on environment-friendly behavior, namely, social class influences environmental friendly behavior by positively influencing happiness.
\end{abstract}

\section{社会阶层、幸福感与环境友好行为}

\author{
叶楠 ${ }^{1, a}$, 张潇 $2, b^{*}$, 余航 ${ }^{3, c}$ \\ 1, 2, 3 江苏师范大学商学院, 徐州, 江苏, 中国 \\ anye@jsnu.edu.cn, b244785316@qq.com, c1174607211@qq.com \\ *通讯作者
}

关键词：社会阶层；幸福感；环境友好行为；CGSS

摘 要: 本文基于中国综合社会调查 (CGSS) 2013年数据, 分析研究主观社会阶层对环境友好 行为之间的影响, 并引入幸福感这一中介变量来解释主观社会阶层对环境友好行为影响的机 制。研究发现，社会阶层正向影响环境友好行为，即正向影响公域的环境友好行为和私域的 环境友好行为。幸福感正向影响私域环境友好行为, 但负向影响公域环境友好行为。此外, 幸福感在社会阶层与环境友好行为的影响中起到部分中介作用，社会阶层通过正向影响幸福 感来影响环境友好行为。

\section{1. 引言}

随着我国生态文明建设的推进，环境问题的关注度越来越高，如何提高大众的环境行为水 平成为众学者研究的重点。环境行为的影响因素总的来说是多方面的。这其中有从人口统计 特征角度进行研究, 诸如性别、年龄、民族、宗教等方面; 还有学者从个体层面进行研究, 诸如大众的环境认知、环境知识、环境责任感等对环境行为的影响。还有学者从社会经济地 位的角度来研究对环境友好行为的影响。总的来说, 社会阶层越高, 社会经济地位越高, 其 环境行为的水平也会越高。为了进一步探讨社会阶层对环境友好行为的影响机制, 本文基于 
CGSS2013 年数据, 并引入幸福感作为中介变量, 来研究社会阶层对环境友好行为的影响。

\section{2. 文献综述与研究假设}

\section{1 社会阶层与环境友好行为}

对于社会阶层的划分, 通常分为主观社会阶层和客观社会阶层。客观社会阶层通常由收 入、教育、职业等因素决定 ${ }^{[1]}$ 。但具体的划分标准并没有达成共识。而主观社会阶层则由个 体的主观判断决定, 个体会出于对自身的认识而做出对自己所处的社会阶层一定的判断。

一般来说, 社会阶层越高, 其教育水平、职业水平和收入水平也会越高, 其总体素质会相 比低阶层的居民高, 他们总体上会对环境保护有一个深刻的认识, 因此他们会进行更多的环 境友好行为。时立荣等 (2016) 基于 CGSS2010 年数据, 从三个方面划分阶层来研究阶层对 环境行为的影响 ${ }^{[2]}$ 。研究表明, 职业阶层地位越高, 居民的环境行为水平越高; 教育阶层地位 越高, 居民的环境行为水平越高; 收入阶层地位越高, 居民的环境行为水平越高。焦开山(2014) 通过 CGSS2010 年数据验证社会经济地位和环境保护行为的关系。其研究表明, 社会经济地 位对环境保护行为有着显著的正向影响, 且处于较高社会经济地位的群体表现出更多的环境 保护行为 ${ }^{[3]}$ 。李娜 (2016) 根据 CGSS2013 年数据实证表明, 社会经济地位会显著影响环境行 为, 其中收入越高, 受教育程度越高, 环境行为水平也越高 ${ }^{[4]}$ 。根据马斯洛的需求理论, 只有 满足人最基本的物质需求之后, 才会开始关注更高精神层面的追求，例如环境保护行为等。 基于此，本文提出假设:

$\mathrm{H} 1$ :社会阶层正向影响环境友好行为。

H1a：社会阶层正向影响公域环境友好行为。

$\mathrm{H} 1 b$ ：社会阶层正向影响私域环境友好行为。

\section{2 幸福感与环境友好行为}

幸福感问题是近年来诸多学者研究的重要问题。葛腾飞等 (2016) 基于 CGSS2013 年数据 研究表明受教育程度能够显著影响居民幸福感, 即受教育程度越高, 居民幸福感越强 ${ }^{5}$ 。黄嘉 文（2013）通过 CGSS2005 年数据研究表明, 受教育程度通过影响收入水平间接影响环境友 好行为, 也就是说受教育程度越高, 其收入水平也越高, 在此基础上, 也会进行更多的环境 友好行为 ${ }^{[6]}$ 。曹大宇 (2009) 研究表明, 社会地位越高, 幸福感水平也就越高 ${ }^{[7]}$ 。六楠楠 (2017) 通过 CGSS2013 年数据研究表明, 幸福能够正向影响居民环境行为。居民幸福感的提升能够 影响居民的环境关心水平, 从而影响居民环境友好行为 ${ }^{[8]}$ 。一般来说, 只有具备一定的社会经 济基础和社会地位, 居民的幸福感程度也会随之提高, 在居民幸福感提升之后, 会从事更多 的精神层面的追求, 同样也会进行更多的环境友好行为。因此, 本文提出假设:

$\mathrm{H} 2$ : 幸福感能够正向影响环境友好行为。

$\mathrm{H} 2 \mathrm{a}$ : 幸福感正向影响公域环境友好行为。

$\mathrm{H} 2 b$ : 幸福感正向影响私域环境友好行为。

H3：幸福感在社会阶层对环境友好行为的影响中起到中介作用。

$\mathrm{H} 3 \mathrm{a}$ ：幸福感在社会阶层对公域环境友好行为的影响中起到中介作用。

$\mathrm{H} 3 \mathrm{~b}$ ：幸福感在社会阶层对私域环境友好行为的影响中起到中介作用。

\section{3. 数据和变量}

\section{1 数据来源及变量测量}

本文所选用的数据来自 2013 中国综合社会调查（CGSS），始于 2003 年，是全国性、综 合性、连续性学术调查项目。CGSS 采用多阶分层概率抽样, 系统、全面地收集社会、社区、 家庭、个人多个层次的数据, 其数据覆盖中国大陆省级行政单位, 在预测消费者行为方面具 有较高的权威性。剔除掉无效数据及缺失数据, 共得到 8311 份有效数据。 
（1）因变量为环境友好行为, 由问卷 “在过去的年里, 您是否从事过下列活动或行为? ” 中的 10 个题项测量。借鉴前人的做法, 本文通过因子分析, 将环境友好行为分为私域友好行 为和公域友好行为。其中，私域环境友好行为由垃圾分类放; 与自己的亲戚朋友讨论环保问 题; 采购日常用品时,自己带购物篮或购物袋; 对塑料包装袋进行重复利用; 主动关注广播,电 视和报刊中报道的环境问题和环保信息，对应问卷的第 1、2、3、4、6 题项。公域环境友好 行为由为环境保护捐款; 积极参加政府和单位组织的环境宣传教育活动; 积极参加民间环保 团体举办的环保活动; 资费养护树林或绿地; 积极参加要求解决环境问题的投诉上诉, 对应 问卷中的第 5、7、8、9、10 题项。由回答者在 “从不” “偶尔” “经常” 中选择，并分别赋 值 1、2、3 分。本文分别将公域和私域友好行为的五个题项的得分进行加总并取均值, 得到 每个受访者公域和私域友好行为的得分。得分越高, 表示进行的环境友好行为越多。

（2）解释变量为社会阶层和幸福感。本文中将社会阶层由题项 “您认为, 您目前处于什 么阶层” 测量, 1 为最底层, 10 为最高层, 由测试者主观回答。另一解释变量幸福感由 “总 的来说，您觉得您幸福吗”的五级量表测量，1 表示非常不幸福，5表示非常幸福。

（3）控制变量。在以往文献的研究中，影响环境友好行为的影响因素有很多，本文总结 以往文献的研究, 将性别、年龄、民族、宗教、收入、教育、城乡、健康、政治面貌、婚姻作 为控制变量。

\section{2 变量描述性统计}

因变量为总的环境友好行为, 其平均值为 1.52 , 其中私域环境友好行为的平均值达到 1.85 , 明显超过平均水平, 而公域环境友好行为的平均值只有 1.19 , 明显低于平均水平, 这说明大 家更多的从事私域的环境友好行为，而更少的从事公域环境友好行为。

解释变量为社会阶层和幸福感。其中社会阶层的平均值为 4.35 , 低于平均水平 5 , 说明大 家对自己社会地位的认可度较低; 幸福感的平均值为 3.76, 高于中等水平 3 , 说明大家对自己 的生活相对满意, 比较幸福。

\section{4. 实证分析}

本文采用 OLS 回归, 分别以总的环境友好行为、公域环境友好行为及私域环境友好行为 作为因变量, 采用层级回归分析考察社会阶层和幸福感对环境友好行为的影响, 及幸福感在 社会阶层和环境友好行为的影响中的中介效应。表 1 是相关性因变量和自变量的相关性分析, 从表 1 可以看出, 主观社会阶层与公域环境友好行为和私域环境友好行为显著相关, 幸福感 和私域环境友好行为显著相关。

接下来进行分层回归分析。第一步将社会阶层纳入模型, 分析社会阶层对环境行为的影 响; 第二步将幸福感作为因变量, 分析社会阶层对幸福感的影响; 第三步分别将社会阶层与 幸福感纳入模型。见表 2 。 
表 1 相关性分析

\begin{tabular}{cccc|c}
\hline & 公域 & 私域 & 社会阶层 & 幸福感 \\
\hline 公域 & 1 & & & \\
\hline 私域 & $0.395^{* * *}$ & 1 & & \\
\hline 社会阶层 & $0.128^{* * *}$ & $0.145^{* *}$ & 1 & \\
\hline 幸福感 & 0.013 & $0.080^{* * *}$ & $0.276^{* * *}$ & \\
\hline
\end{tabular}

***. 在 0.01 级别（双尾）, 相关性显著。

表2 层级回归表

\begin{tabular}{|c|c|c|c|c|c|c|c|c|c|c|}
\hline \multirow{2}{*}{\multicolumn{2}{|c|}{ 被解释变量 }} & M1 & M2 & M3 & M4 & M5 & M6 & M7 & M8 & M9 \\
\hline & & 总行为 & 幸福感 & 总行为 & 公域行为 & 幸福感 & 公域行为 & 私域行为 & 幸福感 & 私域行为 \\
\hline \multirow{10}{*}{$\begin{array}{c}\text { 控制变 } \\
\text { 量 }\end{array}$} & 性别 & $-.052 * * *$ & $-.085 * * *$ & $-.051 * * *$ & .002 & $-.085^{* * *}$ & .001 & $-.105^{* * *}$ & $-.085 * * *$ & $-.104 * * *$ \\
\hline & 年龄 & $.001^{* *}$ & $.005^{* * *}$ & $.001^{* *}$ & $-.001 *$ & $.005 * * *$ & -.001 & $.002 * * *$ & $.005 * * *$ & $.002 * * *$ \\
\hline & 收入 & $.033 * * *$ & $.043 * * *$ & $.033 * * *$ & $.019 * * *$ & $.043 * * *$ & $.019 * * *$ & $.047 * * *$ & $.043 * * *$ & $.046^{* * *}$ \\
\hline & 教育 & $.019 * * *$ & -.002 & $.019 * * *$ & $.011 * * *$ & -.002 & $.011 * * *$ & $.026^{* * *}$ & -.002 & $.027 * * *$ \\
\hline & 民族 & -.017 & $-.074 *$ & -.017 & .015 & $-.074 *$ & .014 & $-.049 * *$ & $-.074^{*}$ & $-.047 * *$ \\
\hline & 宗教 & .016 & .048 & .016 & $.035^{* *}$ & .048 & $.035 * *$ & -.003 & .048 & -.004 \\
\hline & 党员 & $.077 * * *$ & $.129^{* * *}$ & $.077 * * *$ & $.080^{* * *}$ & $.129^{* * *}$ & $.082 * * *$ & $.074 * * *$ & $.129 * * *$ & $.072^{* * *}$ \\
\hline & 城乡 & $.102 * * *$ & $-.114 * * *$ & $.103^{* * *}$ & $.033^{* * *}$ & $-.114 * * *$ & $.031 * * *$ & $.172^{* * *}$ & $-.114 * * *$ & $.174^{* * *}$ \\
\hline & 健康 & $.007^{*}$ & $.155^{* * *}$ & .007 & .004 & $.155^{* * *}$ & .006 & $.010^{*}$ & $.155^{* * *}$ & .007 \\
\hline & 婚姻 & -.024 & .041 & -.024 & -.023 & .041 & -.022 & -.025 & .041 & -.026 \\
\hline \multirow{5}{*}{$\begin{array}{c}\text { 解释变 } \\
\text { 量 }\end{array}$} & $\begin{array}{c}\text { 社会阶 } \\
\text { 层 }\end{array}$ & $.010^{* * *}$ & $.114 * * *$ & $.010^{* * *}$ & $.011 * * *$ & $.114 * * *$ & $.012 * * *$ & $.009 * *$ & $.114 * * *$ & $.007^{*}$ \\
\hline & 幸福感 & & & .004 & & & $-.013 * *$ & & & $.020^{* * *}$ \\
\hline & R2 & 0.199 & 0.118 & 0.199 & 0.089 & 0.118 & 0.089 & 0.196 & 0.118 & 0.197 \\
\hline & 调整R2 & 0.198 & 0.117 & 0.198 & 0.087 & 0.117 & 0.088 & 0.195 & 0.117 & 0.196 \\
\hline & F值 & 187.194 & 101.102 & 172.200 & 73.288 & 101.102 & 67.970 & 184.297 & 101.102 & 170.066 \\
\hline
\end{tabular}

表 2 中, 模型 $1 、 4 、 7$ 考察自变量对因变量的影响。模型 $1 、 4 、 7$ 表明, 主观社会等级 均正向影响环境友好行为 $(M 1, \beta=0.010, p<0.001 ; M 4, \beta=0.011, p<0.001 ; M 7, \beta=0.009, p<0.01$ ), 即认为自己处在更高阶层的人会从事更多的公域和私域环境友好行为, 假设 $\mathrm{H} 1$ 成立; 模型 3、6、9 表明, 幸福感虽然对总的环境友好行为影响不显著 $(M 3, \beta=0.004, p>0.05)$, 但显著影 响公域和私域环境友好行为。具体来看, 幸福感负向影响公域环境友好行为 $(\mathrm{M} 6, \beta=-$ $0.013, \mathrm{p}<0.01)$, 正向影响私域环境友好行为( M9, $\beta=0.020, p<0.001)$, 假设 $\mathrm{H} 2 \mathrm{a}$ 不成立, 假设 $\mathrm{H} 2 \mathrm{~b}$ 成立。这说明，越幸福的人会从事更多的私域友好行为，而更少的从事公域环境友好行为。

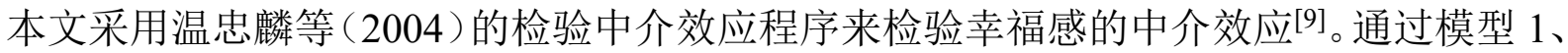
2 可以看出, 社会阶层均正向显著影响总的环境友好行为和幸福感, 模型 3 将社会阶层和幸 福感同时纳入模型, 社会阶层仍显著影响总的环境行为, 说明幸福感在社会阶层对总的环境 
友好行为中起到部分中介作用。通过模型 4、5 可以看出, 社会阶层和幸福感显著正向影响公 域环境友好行为, 模型 6 表明幸福感在社会阶层对公域环境行为的影响中起到部分中介作用。 同样通过模型 7、8、9 可以看出社会阶层在幸福感对私域环境友好行为的影响中起到部分中 介作用，因此假设 H3 成立。

\section{5. 总结}

本文基于 CGSS2013 数据探讨社会阶层、幸福感和环境友好行为之间的关系，并得出以 下结论：社会阶层正向影响环境友好行为, 即正向影响公域环境友好行为和私域环境友好行 为。对自己所处阶层评价越高的人会进行更多的环境友好行为; 幸福感显著影响环境友好行 为, 但幸福感仅正向影响私域环境友好行为, 而负向影响公域环境友好行为。对自我评价越 幸福的人越会进行更多的私域环境友好行为。幸福感在社会阶层对环境友好行为的影响中起 到部分中介作用。即社会阶层通过影响幸福感来影响环境友好行为。

本文通过实证证明社会阶层对环境行为有显著的正向影响, 因此, 我们需要不断完善社会 阶层结构。首先我们需要弱化家庭、户口等先赋性因素构建阶层地位的获取机制; 然后不断 强化教育所带来的社会地位提升的优势。考虑到社会阶层较低的群体一定程度上是因为受教 育程度低导致环境保护意识薄弱, 因此加强对其环境保护知识的宣传也是很有必要的。提升 大众的受教育程度, 增加其收入水平一定程度上可以提升社会阶层, 只有满足社会阶层的提 升，才有进一步的精神层面的需求，因此也会进行更多的环境友好行为。

对于政府而言，应该不断缩小贫富差距，使得社会阶层的差异缩小。并且应该加快经济 增长和居民收入水平，尽量提升居民的受教育程度，普及更多的环境知识，提升居民对环境 问题的认知水平，加强居民环保意识，提升居民生活幸福感。

\section{致谢}

本文为国家自然科学基金项目 《规范诉求对可持续消费行为的影响机理研究：基于社会 责任认知视角》（项目批准号：71502072）、江苏省研究生科研与实践创新计划项目《社会 规范对可持续消费行为的影响研究》（项目编号：KYCX17_1610）的阶段性成果之一。

\section{References}

[1] Liu Jingming, Li Lulu. Stratification: living space, life style, social interaction and stratum identification -- An Empirical Study of the social stratification in China's urban society: [J]. sociology research, $2005(03): 52-81+243$.

[2] Li Rong, Chang Liang, Yan Hao. Analysis of the stratum differences of environmental behavior - An Empirical Analysis Based on China's comprehensive social survey in 2010 [J]. Shanghai Institute of administration, 2016, 17 (6): 78-89.

[3] Jiao Kaishan. Social economic status, environmental awareness and environmental protection behavior -- an analysis based on structural equation model [J]. Inner Mongolia Social Science (Chinese version), 2014, 35 (6): 138-144.

[4] Li Na. Socioeconomic status and environmental behavior difference between urban and rural residents: Based on the empirical data of CGSS2013, [J]. Sichuan environment, 2016, 35 (6): 132-136.

[5] Ge Tengfei, Chen Yingying, Zhou Yanfang, etc.. Education, health and well-being of residents - An Empirical Analysis Based on CGSS2013 [J]. Journal of Suzhou University, 2016, 31 (2): 53-56. 
[6] Huang Jiawen. Education level, income level and happiness of urban residents in China: An Empirical Analysis Based on CGSS2005, [J]. society, 2013, 33 (5): 19-20.

[7] Cao Dayu. Empirical analysis of class differentiation, social status and subjective well being [J]. statistics and decision making, 2009 (10): 89-91.

[8] Kang Nan Nan, Wang Er da. Influence of SWB on environmental behavior of residents: [J]. statistical study, 2017, 34 (5): 82-93.

[9] Wen Zhonglin, Zhang Lei, Hou Jie Tai, et al. Mediating effect test procedure and its application [J]. psychological journal, 2004, 36 (5):614-620. 